\title{
Análisis y valoración del modelo inclusivo en los sistemas de apoyo de centros de infantil y primaria
}

\section{Analysis and evaluation of the inclusive model in infant and primary centre support systems}

\author{
María José Nadal Morant ${ }^{1}$ \\ Maria.Jose.Nadal@uv.es \\ Claudia Grau Rubio \\ Claudia.Grau@uv.es \\ José Peirats Chacón \\ Jose.Peirats@uv.es \\ Universidad de Valencia, España
}

\section{Resumen:}

En esta investigación se analiza el funcionamiento y la organización de los sistemas de apoyo utilizados en centros de Educación Infantil y Primaria de la Comunidad Valenciana. Planteamos hasta qué punto los sistemas de apoyo en estos centros contribuyen a mejorar la organización de un sistema inclusivo que resulte accesible para todos. En el estudio de casos han participado 24 profesionales pertenecientes a cuatro centros que tienen diferentes modalidades de escolarización. Para la recogida de información, se han manejado instrumentos cualitativos como son la entrevista, la observación y el análisis documental. Según los datos obtenidos, los sistemas de apoyo no se ajustan de forma integral a enfoques inclusivos. Se refleja, en el análisis efectuado, una aproximación en algunos elementos relacionados con su organización. Por otra parte, existen otros elementos que todavía se con-

\begin{abstract}
:
The functioning and organization of support systems in Infant and Primary Education Centres in the Valencian Community are studied in this study. Specifically, this research looks at the question of to what degree these centres contribute to the provision of an inclusive system which is accessible for everyone. Twenty-four professionals from four different centres, all of which use different schooling methods, participated in this study. To collect the data, qualitative methods, such as interviews, observation and analysis of documents, were used. According to the data obtained, the support systems are not comprehensively tailored towards inclusivity. The study carried out showed that certain elements of organization of inclusivity are present. However, there are other elements that remain far from the standards of inclusivity required in modern schooling, among which we can find the type of
\end{abstract}

1 Dirección para correspondencia (correspondence address):

María José Nadal Morant. Dpto. de Didàctica i Organització Escolar. Facultat de Magisteri. Universidad de Valencia. Avenida Blasco Ibáñez, 30. 46010 Valencia (España). 
Análisis y valoración del modelo inclusivo en los sistemas de apoyo de centros de infantil y primaria

María José Nadal Morant, Claudia Grau Rubio y José Peirats Chacón

sideran alejados de posiciones inclusivas, entre ellos: el tipo de alumnado que recibe apoyo educativo, el lugar o espacio donde los alumnos lo reciben o el modo de agrupamiento que se establece. Como conclusión, a pesar de que se observa un cierto avance en el modelo de apoyo, todavía existe una concepción tradicional centrada en el alumnado.

\section{Palabras clave:}

Organización escolar; sistemas de apoyo; educación inclusiva; necesidades educativas especiales; modalidades de escolarización. student who receives educational support; the place in which this support is given and the way in which the students are grouped together. In conclusion, in spite of obvious advances in the support model for inclusivity, there is still an extremely traditional concept of this support focused principally on the students.

\section{Key words:}

School organization; support systems; inclusive education; special educational needs; schooling methods.

\section{Résumé:}

Dans cette étude, nous analysons le fonctionnement et l'organisation des systèmes de soutien, utilisés dans les Centres d'Éducation Primaire de la Communauté Valencienne. Nous démontrons à quel point les systèmes de soutien de ces centres contribuent à améliorer l'organisation d'une école inclusive qui soit accessible à tous. Dans le cadre de cette recherche, nous avons bénéficié de la participation de 24 professionnels provenant de 4 centres qui possèdent différentes modalités de scolarisation. Afin de recueillir l'information, nous avons utilisé des instruments qualitatifs tels que l'entretien, l'observation et l'analyse documentaire. D'un côté, d'après les données obtenues, les systèmes de soutien ne s'adaptent pas complètement aux approches inclusives. Dans cette analyse, on reflète un rapprochement de quelques éléments relatifs à l'organisation. D'un autre côté, il existe d'autres éléments qui sont encore considérés étrangers aux positions inclusives. Parmi ceux-là, les types d'élèves qui bénéficient du soutien éducatif, le lieu ou l'espace où les élèves le reçoivent, ou bien le regroupement que l'on établit. Pour conclure, malgré l'observation d'un certain progrès du modèle de soutien, il existe encore une conception traditionnelle centrée sur l'effectif scolaire.

\section{Mots clés:}

Organisation scolaire; systèmes de soutien; éducation inclusive; besoins éducatifs spéciaux; modalités de scolarisation.

Fecha de recepción: 27-7-2016

Fecha de aceptación: 5-10-2016

\section{Introducción}

Vivimos en una sociedad cada vez más diversa. Entre las principales finalidades del sistema educativo se encuentran la de eliminar los procesos de exclusión generados desde los ámbitos sociales y dar respuesta a la diversidad. El modelo educativo que permite gestionar con equidad y atender realmente a la diversidad ha de ser inclusivo, en el cual el alum- 
nado con discapacidad obtenga igualdad de oportunidades (Muntaner, 2010). En estas páginas resaltamos que la presencia del alumnado con necesidades educativas especiales (NEE) en las escuelas es un derecho fundamental en nuestro sistema educativo. Compartimos, por tanto, el reconocimiento y la valoración de la educación como un derecho esencial que ha de garantizarse a todas las personas, sin ningún tipo de discriminación (Escudero, 2012; Escudero y Martínez, 2011).

Este planteamiento nos incita a exponer un conjunto de interrogantes en relación a cómo se están desarrollando actualmente las prácticas inclusivas en nuestros centros, y qué posibilidades reales existen para alcanzar una auténtica y verdadera inclusión. Nuestra finalidad, por tanto, se dirige a analizar los sistemas de apoyo que se desarrollan en esos centros. Para alcanzar este propósito es necesario considerar la organización de los centros como un elemento fundamental para dar respuesta a la diversidad (AAIDD, 2011; Giné, 2001; Torres, 2012). En este sentido, el último autor señala que "la atención a la diversidad se incluye dentro de un enfoque educativo global que impregna todos los elementos de la escuela, donde la organización escolar desempeña una función instrumental para facilitar su puesta en práctica" (p. 56). Pretendemos, por tanto, desvelar, analizar e interpretar las variaciones en la estructura organizativa de los sistemas de apoyo de cuatro centros para tratar de impulsar procesos de cambio hacia la inclusión educativa.

Para conseguir este objetivo, en primer lugar, nos detenemos en el concepto de la educación inclusiva. En este punto Sandoval, Simón y Echeita (2012, p. 120) sostienen que es "el proceso vinculado a la transformación profunda de los sistemas educativos establecidos, de modo que la educación formal (y no formal) se promueva y asegure la defensa de la dignidad e igualdad de todos los alumnos, como derechos inalienables, desde el reconocimiento y respeto de su diversidad". A la que sumamos también los principios y las características de las escuelas inclusivas que han aportado autores como Arnaiz (1996, 2003 y 2011), Moriña (2004), Muntaner (2010), Parrilla (1992) o Stainback y Stainback, (1992 y 1999) entre otros, sin olvidar aquellas directrices y criterios que dispone la legislación sobre la educación inclusiva desde diversos organismos internacionales, nacionales y autonómicos.

En segundo lugar, hay que señalar las reflexiones que realizan algunos autores como AAIDD (2011), Booth y Ainscow (2002), Nieto (1999), Parrilla (1996), Sandoval, Simon y Echeita (2012) Stainback y Stainback 
Análisis y valoración del modelo inclusivo en los sistemas de apoyo de centros de infantil y primaria

María José Nadal Morant, Claudia Grau Rubio y José Peirats Chacón

(1992), entre otros, acerca del concepto de apoyo educativo y las características más significativas del enfoque inclusivo, así como los diferentes modelos propuestos por distintos autores. En síntesis, el concepto de apoyo ha ido evolucionando en nuestro sistema educativo en función de los cambios que se han producido en el ámbito disciplinar de la educación especial y en las distintas concepciones de la diferencia, que han ido progresivamente desde los enfoques deficitarios hasta los enfoques de carácter cultural-integrador (Torres, 2004).

Además, hay que tener en cuenta el apoyo interno, entendido como "la respuesta desde el propio centro a sus necesidades educativas, contando para ello con los elementos materiales, estructuras organizativas y elementos personales necesarios" (Moya, 2002, p. 13). Para esta autora, el apoyo interno puede intervenir desde distintos puntos de vista y haciendo referencia a diferentes modalidades, según las características de los propios profesionales o de los contextos y situaciones. Teniendo en cuenta las funciones de los apoyos internos, Lozano (2007), Monzón (2011), Moya (2002) y Parrilla (1996) distinguen los siguientes modelos de apoyo interno: apoyo terapéutico, apoyo colaborativo-individual, apoyo consulta y apoyo curricular, que surgen de dos factores: sobre quién recae el apoyo (sobre el individuo / alumnado o sobre el currículo / escuela / programa / proyecto / profesorado) y el modo en que el profesional de apoyo interactúa con el profesorado ordinario (como experto o de modo colaborativo).

Si nos fijamos en los diferentes tipos de apoyo interno podemos observar que, frente al modelo de apoyo terapéutico que surge como consecuencia de un problema que tiene exclusivamente el alumnado, y donde es necesaria la intervención del profesorado especialista que intenta proponer formas de aprender diferentes $y$, habitualmente, segregadas del currículo ordinario, nos encontramos, en el otro extremo, el modelo de apoyo curricular, el cual concibe que el profesorado ordinario es el responsable del cambio, considerándole capaz de aprender a responder a las necesidades que se van derivando de las prácticas educativas cotidianas y entendiendo que su trabajo en ellas favorece la mejora de las prácticas inclusivas.

En este sentido, hay autores como Arnaiz (2011), Monzón (2011) Muntaner (2010) y Sandoval, Simón y Echeita (2012) que defienden el modelo de apoyo curricular para una educación de calidad con equidad, el cual hemos tomado como referencia en nuestro estudio. Por su parte, 
Arnaiz (2011) apunta que el modelo curricular parte de la idea de que todos los alumnos y alumnas aprenden de forma diferente, por lo que los centros han de adaptarse a esas diferencias desarrollando modelos que sepan responder a las variadas necesidades que presenta el alumnado. Supone, por tanto, un cambio en la organización y en las estrategias de enseñanza y aprendizaje que se desarrollan en las escuelas, con el fin de que se garantice el ejercicio de los principios de igualdad y equidad a los que todo ser humano tiene derecho. Siguiendo en la misma línea, Muntaner (2010) propone un modelo de apoyo generalizado dirigido al conjunto de la escuela, que trabaja con todo el profesorado para compartir y buscar conjuntamente las alternativas educativas que permitan diversificar y aceptar el currículo para todo el alumnado, desde una perspectiva contextual no desde la perspectiva individual-terapéutica.

En cuanto a la situación actual de los apoyos en nuestro contexto, es necesario conocer en profundidad la normativa autonómica que regula los sistemas de apoyo en los centros de infantil y primaria, centrándonos en el análisis de los elementos organizativos que intervienen en los sistemas de apoyo. Lo que nos ayudará a describir la situación real regulada por las disposiciones legales, y facilitará delimitar el modelo de apoyo que predomina hoy en nuestros centros.

\section{Método}

Optamos por la metodología cualitativa, situada en la perspectiva humanística, para una investigación realizada desde dentro, donde adquiere un papel fundamental lo individual y lo subjetivo (Abalde y Muñoz, 1992). Parte del supuesto de que los actores sociales no son meros objetos de estudio, sino que tienen un significado, hablan, reflexionan sobre su situación y toman decisiones.

En consecuencia, nuestro estudio de tesis se encuadra dentro de la corriente de investigación descriptiva y de análisis de la práctica de los profesionales que ejercen apoyo al alumnado con discapacidad en los centros. Se trata de un tipo de indagación caracterizado por ser "un proceso de investigación, un camino para adentrarse en el estudio de la vida humana, describiendo y reconstruyendo las escenas culturales de grupos de personas, los contextos, actividades y opiniones de los participantes" (Coronel, 1998, p. 234). 
Análisis y valoración del modelo inclusivo en los sistemas de apoyo de centros de infantil y primaria

María José Nadal Morant, Claudia Grau Rubio y José Peirats Chacón

\section{Objetivos}

Se analizan los sistemas de apoyo desde un planteamiento inclusivo. Para ello, realizamos un estudio de la organización de los elementos de los apoyos que intervienen en los centros. Concretamente, han sido el tipo de alumnado que recibe apoyo educativo, las personas que lo proporcionan, los materiales y recursos didácticos utilizados, el lugar o espacio donde reciben los apoyos, la frecuencia con que se proporcionan, el modo de agrupamiento que se establece, el tipo de adaptaciones que se realizan y el tipo de actuaciones de los diferentes profesionales que intervienen. En este trabajo, establecemos dos objetivos a alcanzar:

1. Valorar cuál es el nivel de aproximación de cada uno de los elementos seleccionados hacia las prácticas inclusivas en las aulas.

2. Establecer el modelo de apoyo que predomina en los centros analizados, considerando como punto de partida el modelo inclusivo.

\section{Descripción de la muestra}

Se han seleccionado cuatro centros de infantil y primaria que presentan diferentes modalidades de escolarización, según establece la normativa vigente en la Comunidad Valenciana: un centro ordinario, un centro con aula de Comunicación y Lenguaje $(\mathrm{CyL})$, un centro de atención preferente y un centro con unidades específicas de Educación Especial.

En primer lugar, seleccionamos el centro ordinario por ser un centro que acoge a la diversidad del alumnado. Se escolarizan alumnos con diferentes necesidades y está dotado de recursos personales y técnicos adecuados dentro de un contexto normalizado e integrador. Suelen ejercer apoyo profesorado de Pedagogía Terapéutica (PT), de Audición y Lenguaje $(\mathrm{AL})$, educador/a de educación especial y el orientador/a. El alumnado con NEE recibe apoyo en las aulas de PT y AL o bien en el aula ordinaria, con una duración variable regulada por la normativa vigente y sigue el desarrollo del currículo ordinario con la aplicación de medidas de adaptación curricular o refuerzo educativo.

En segundo lugar, el centro con aula de Comunicación y Lenguaje. Es una unidad específica de educación especial ubicada en centro ordinario, con carácter experimental que intenta dar una respuesta educativa adecuada al alumnado con trastorno Mixto del Lenguaje ReceptivoExpresivo (TML) o con Trastorno del Espectro Autista (TEA). Dispone de 
un espacio físico apropiado e integrado en el centro ordinario, donde el alumnado recibe la mayor parte de los apoyos, aunque existen diferentes niveles de inclusión en las aulas ordinarias, en función de las necesidades del alumnado y mediante la organización de horarios personalizados. Es atendida, exclusivamente, por profesorado de PT, de AL y un educador/a y suele utilizar programas y metodologías específicas para trabajar el lenguaje y la comunicación.

El tercer caso es un centro de atención preferente, donde se da las condiciones de escolarización para alumnado con diferentes necesidades educativas especiales, según la Orden de 16 de julio de 2001. Son centros con dotaciones y equipamientos singulares o una especialización profesional difícilmente generalizable. En el centro analizado se encuentra alumnado con discapacidad motriz que recibe apoyo en las aulas de PT y AL y en las aulas ordinarias de forma puntual. Dispone de dos maestras de PT, una maestra de AL, tres educadoras, dos fisioterapeutas y el orientador. Se intenta que el alumnado permanezca el máximo tiempo posible en el aula ordinaria con los apoyos necesarios.

Finalmente optamos por el centro con unidades específicas. Suelen ser centros en poblaciones alejadas de los centros específicos, que disponen de aulas de educación especial a tiempo completo o parcialmente completo, para alumnado con adaptaciones significativas del currículo. El centro seleccionado escolariza, mayoritariamente, a alumnado con discapacidad auditiva y, para proporcionar los apoyos, dispone de una maestra de PT, un equipo de cuatro maestras de $\mathrm{AL}$, una educadora de educación especial y el orientador, quienes atienden exclusivamente a este alumnado con programas y metodologías específicas.

Ha sido un total de 16 maestros/as, 4 orientadores/as y 4 integrantes del equipo directivo de centros públicos ubicados en diferentes localidades de la Comunidad Valenciana. Centros formados por entre 10-18 unidades, que integran alrededor de 250-400 alumnos y alumnas, con una plantilla de profesorado comprendida entre 20 y 30 profesionales.

\section{Instrumentos de recogida de datos}

En el seno de la metodología cualitativa, hemos seleccionado el estudio de caso, es decir, "el estudio de la particularidad y de la complejidad de un caso singular, para llegar a comprender su actividad en circunstancias importantes" (Stake, 1998, p. 11). La investigación sobre las prácticas in- 
Análisis y valoración del modelo inclusivo en los sistemas de apoyo de centros de infantil y primaria

María José Nadal Morant, Claudia Grau Rubio y José Peirats Chacón

clusivas es un proceso construido socialmente y conviene ser estudiado desde situaciones reales y problemas singulares dentro de contextos estructurales y situacionales.

Utilizamos, por ello, el estudio de casos como técnica de trabajo a través del análisis de cuatro tipos de centros. En la clasificación propuesta por Stake (1998), esta investigación es un estudio colectivo de centros, pero sobre todo es instrumental al centrarse en los sistemas de apoyo de los casos estudiados. Para la recogida de los datos se han utilizado instrumentos diferentes como son la entrevista, la observación y el análisis documental.

En primer lugar, se realizaron entrevistas a los profesionales implicados en cada uno de los centros de estudio que intervienen, directa o indirectamente, en los apoyos. Es decir, se realizaron "reiterados encuentros cara a cara entre el investigador y los informantes, (...) dirigidos hacia la comprensión de las perspectivas que tienen respecto de sus vidas, experiencias o situaciones, tal como las expresan con sus propias palabras" (Taylor y Bogdan, 1996, p. 101). Las entrevistas tuvieron lugar en las propias aulas del profesorado de forma espontánea, no artificial, en un ambiente distendido y de confianza.

En segundo lugar, hemos utilizado la observación no participante, de la que Goetz y LeCompte (1988) señalan que "consiste, exclusivamente, en contemplar lo que está aconteciendo y registrar los hechos sobre el terreno" (p. 153), en aquellas dependencias de los centros que forman parte del contexto habitual del alumnado y profesorado, y en donde intervienen y desarrollan sus actividades de apoyo.

Otra fuente de información importante nos la ha ofrecido el análisis de los documentos de los centros (PEC, PAD, PCC, PGA, MAC...), a los que hemos podido acceder previa autorización de la dirección. La recogida de documentos institucionales nos ayuda a completar la información y a comprobar el nivel de coherencia entre el contenido de los documentos y los resultados de las entrevistas.

A través de estas tres técnicas, se ha analizado la información referida a los diferentes elementos que intervienen en la organización del proceso educativo de los apoyos. Para el análisis se han organizado los datos de forma coherente y lógica mediante la clasificación y categorización. Lo que nos ha permitido, en función de la literatura consultada y de los objetivos propuestos, darles forma, reelaborarlos e interpretarlos a dos niveles: uno de ellos de carácter descriptivo en cada uno de los elemen- 
tos estudiados y otro comparativo para establecer qué apoyos favorecen o no la inclusión.

\section{Resultados y discusión}

Dos eran los propósitos enumerados en el apartado de método, el primero de ellos se fijaba en el nivel de aproximación hacia las prácticas inclusivas en las aulas, veamos ahora los resultados.

Desde el análisis de los resultados de las entrevistas y de las observaciones, el tipo de intervención-actuación de los apoyos desarrollada en las aulas de los cuatro centros no favorece la inclusión. Las actividades que realiza el alumnado con NEE no tienen ninguna relación con las que realiza el tutor/a en el aula, los materiales suelen ser diferentes, los agrupamientos homogéneos, es decir, se sitúan juntos al lado del maestro de apoyo, sobre todo en primaria diferenciándolos del resto de la clase, o salen al aula de PT. Veamos lo que nos señalaban al respecto una orientadora y una maestra de PT entrevistadas:

Intentamos que sea un agrupamiento más bien homogéneo por edades, (...) y por problemas que tengan... Dentro del aula, a lo mejor, es un niño, o dos niños, entonces se intenta que estén más o menos cerca para que, cuando entre la persona que va a reforzarlos, esté cerca (3EO, p. 4, 104-109).

Los alumnos se atienden en mi clase, o sea, en el aula de PT la mayoría, porque aquí tienes todo el material, además ellos están más centrados aquí y no se distraen, porque en clase la atención también suele estar más dispersa, porque están los compañeros y hay muchos estímulos en general (1EPT p. 3, 69-72).

Los resultados de las entrevistas coinciden, prácticamente, con los obtenidos en las observaciones realizadas en las aulas de infantil, primaria, el aula de PT y el de AL de los cuatro centros. En todos los centros proporcionan apoyo los especialistas ( $\mathrm{PT}, \mathrm{AL}$, de apoyo de infantil y educadora) y profesorado que dispone de tiempo libre en su horario. En relación a las tareas que realiza el apoyo, hemos observado que, en algunas aulas, refuerza al alumnado con NEE dentro del aula, pero las actividades que se realizan no están relacionadas con la actividad que está desempeñando en ese momento el tutor/a (4OEI, 4OEP). 
Análisis y valoración del modelo inclusivo en los sistemas de apoyo de centros de infantil y primaria

María José Nadal Morant, Claudia Grau Rubio y José Peirats Chacón

Sin embargo, la mayoría de los documentos analizados no se ajus$\tan$ a los datos obtenidos en las entrevistas y en las observaciones. Por ejemplo, existen diferencias significativas entre la organización de los apoyos entre la etapa de infantil y primaria. Sin embargo, no se refleja en los documentos así como tampoco existe una coherencia entre el contenido de dichos documentos, referido a las prácticas inclusivas de los apoyos, con la realidad de los centros. También destacamos que, en los documentos analizados, no se refleja la totalidad de la información relativa a la organización de los apoyos. En la mayoría de los centros no especifican los diferentes elementos que intervienen, no están completos y los datos suelen ser muy genéricos. Por otro lado, el Plan de Atención a la Diversidad (PAD), documento donde se establecen las líneas de actuación para atender a la diversidad y las medidas organizativas de los apoyos, no lo tienen elaborado todos los centros, únicamente el ordinario y el de atención preferente.

Por economía de espacio, reflejamos en una tabla una síntesis de los resultados obtenidos en este objetivo:

Tabla 1. Síntesis de resultados sobre la organización del sistema de apoyos.

ELEMENTOS CARACTERÍSTICAS DE LOS APOYOS

El alumnado que recibe apoyo presenta NEE derivadas de disca-

Alumnado que pacidades físicas, psíquicas y sensoriales, TEA, TDAH y dificulrecibe apoyo tades del aprendizaje... En la mayoría de centros, el alumnado que no presenta NEE, no se beneficia de estos recursos.

Los profesionales que proporcionan apoyo suelen ser de PT,

Profesionales $\mathrm{AL}$, de refuerzo en educación infantil, educador/a de educa-

que propor- ción especial, orientador/a y el profesorado que dispone de

cionan apoyo tiempo libre en su horario. Se considera poco significativo el apoyo recibido por los compañeros/as de clase.

Los materiales y recursos didácticos que se utilizan con el alumnado con NEE, suelen ser los mismos que utilizan los

Recursos ma- demás compañeros/as de la clase, como son libros de texteriales to, cuadernos o fichas, pero adaptado a sus necesidades. No obstante, en aquellos alumnos/as que presentan niveles más bajos, se utilizan materiales específicos diferentes al aula.

El lugar o espacio donde el alumnado recibe los apoyos, se elige en función de sus características y de los profesionales

Organización que intervengan. El profesorado que dispone de tiempo libre, espacio suelen intervenir dentro del aula ordinaria, los de PT dentro o fuera del aula ordinaria y los de AL no suelen entrar e intervienen en aulas específicas. 


\begin{tabular}{|c|c|}
\hline $\begin{array}{c}\text { Organización } \\
\text { tiempo }\end{array}$ & $\begin{array}{l}\text { Se refuerza al alumnado con NEE tres o cuatro sesiones sema- } \\
\text { nales, siempre en función de la gravedad sus necesidades y } \\
\text { de los recursos personales que disponga el centro. No obstan- } \\
\text { te, el tiempo de atención está determinado por la normativa } \\
\text { vigente en función del diagnóstico del alumnado. }\end{array}$ \\
\hline $\begin{array}{l}\text { Agrupamien- } \\
\text { tos }\end{array}$ & $\begin{array}{l}\text { El tipo de agrupamiento para recibir el apoyo suele ser indi- } \\
\text { vidual o en pequeños grupos formados por dos, tres o cuatro } \\
\text { alumnos/as, bien sea en el aula ordinaria bien en las aulas } \\
\text { de PT o AL. Existe un criterio de homogeneidad en el tipo } \\
\text { de agrupamiento; es decir, el alumnado se suele agrupar por } \\
\text { características similares, por edades, por problemática o nivel } \\
\text { de conocimientos. El profesorado que ejerce apoyo dentro } \\
\text { del aula ordinaria suele situarse al lado del alumno/a o en la } \\
\text { mesa donde se encuentra el alumnado que necesita apoyo. }\end{array}$ \\
\hline Adap & $\begin{array}{l}\text { Predominan las adaptaciones curriculares frente a las organi- } \\
\text { zativas o de contexto. En la mayoría de los centros el alumna- } \\
\text { do con NEE sigue el mismo currículo que el resto de la clase, } \\
\text { pero adaptándolo a sus necesidades. Se sigue la programa- } \\
\text { ción de aula, se suelen trabajar los mismos contenidos, pero a } \\
\text { un nivel más bajo, y se adaptan las actividades. Se refuerza el } \\
\text { trabajo del aula al alumnado que lo necesita. Únicamente se } \\
\text { utilizan programas diferentes y específicos con el alumnado } \\
\text { con mayores dificultades. }\end{array}$ \\
\hline $\begin{array}{l}\text { Funcio } \\
\text { profesi }\end{array}$ & $\begin{array}{l}\text { Los profesionales que ejercen apoyo trabajan directamente } \\
\text { con el alumnado con NEE de forma individualizada o en pe- } \\
\text { queños grupos, bien sea en el aula ordinaria bien en las aulas } \\
\text { de PT o AL. Por lo general, cuando intervienen dos profesio- } \\
\text { nales en la misma aula (tutor/a y el de PT o cualquier otro) } \\
\text { el tutor/a suele dirigir la clase y las actividades; trabaja con } \\
\text { todo el alumnado de la clase y el de PT o de apoyo ayuda } \\
\text { alumnado con NEE. Además, en algunas aulas en las que in- } \\
\text { terviene el de PT, las actividades que se desarrollan no están } \\
\text { relacionadas con la actividad que está desempeñando en ese } \\
\text { momento el tutor. }\end{array}$ \\
\hline
\end{tabular}

El segundo de los objetivos trataba de establecer el modelo de apoyo que predomina en los centros analizados, teniendo en cuenta el modelo inclusivo. Al respecto señalamos que es necesario replantear la organización y el funcionamiento de los apoyos en coherencia con el modelo de apoyo inclusivo, ya que tal y como se organicen los apoyos en los centros se pueden favorecer o no las prácticas inclusivas (Parrilla, 1996; Arnaiz, 2003). Veamos, ahora, los resultados encontrados en los elementos analizados. 
Análisis y valoración del modelo inclusivo en los sistemas de apoyo de centros de infantil y primaria

María josé Nadal Morant, Claudia Grau Rubio y José Peirats Chacón

Si nos fijamos en el alumnado que recibe apoyo, se observa que son tratados como diferentes lo que comporta implicaciones educativas de carácter restrictivo y segregador. En el centro con aula CyL, el de atención preferente y el centro con aulas específicas, el alumnado que recibe apoyo presenta discapacidades muy concretas, y cada uno de estos centros se especializa en un tipo de discapacidad. En cambio, en el ordinario el alumnado que recibe apoyo presenta diferentes tipos de trastornos. Lo que muestran en común los cuatro centros es que sólo se atienden al alumnado con NEE, y el resto no se beneficia de estos recursos. Este planteamiento se contradice con el modelo de apoyo global, que tiene como referente al conjunto del grupo-clase y de la escuela, por lo que cualquier alumno puede precisar apoyos (Arnaiz, 2003; Muntaner, 2010; Moya, 2012).

Si nos centramos en las personas que proporcionan apoyo en los centros, los cuatro centros disponen del mismo tipo de profesionales para ejercer el apoyo, aunque varía en número de recursos en función de la tipología de centro. En este sentido, el centro con aula CyL, el de atención preferente y el centro con aulas específicas disponen de mayor número de recursos respecto al ordinario. Por otra parte, a diferencia de la opinión de Sandoval, Simón y Echeita (2012), quienes defienden que el apoyo lo realiza profesorado específicamente designado al respecto, como son los especialistas en PT o AL; según los resultados alcanzados en los cuatro centros, el apoyo se hace extensible a otros profesionales que trabajan en el centro, como el de refuerzo en infantil, el educador/a y aquellos profesionales que disponen de tiempo libre. En este sentido, si nos centramos en las propuestas inclusivas, los tutores no deben ceder la responsabilidad del alumnado con NEE a los especialistas, sino que deben solicitar su ayuda y colaboración para ampliar su oferta educativa y que todo el alumnado pueda beneficiarse de estas nuevas propuestas didácticas (Muntaner, 2010).

En cuanto a la utilización de materiales y recursos didácticos en los centros por parte del alumnado con NEE. Según los resultados de las observaciones y las entrevistas del centro con aula CyL y del centro con aulas específicas, se utilizan materiales y recursos más específicos en función de las características del alumnado; en cambio, en el centro ordinario y el centro de atención preferente se suelen utilizar los mismos materiales (fichas, libros de texto...) que el resto de compañeros/as, lo cual favorece la inclusión. No obstante, en este aspecto López Melero 
(2011) defiende, en oposición, que las tareas de aprendizaje deben apoyarse en materiales nuevos con funciones diferentes.

El lugar donde reciben los apoyos es diferente en función de la tipología del centro. El alumnado con NEE escolarizados en aula CyL y el de aulas específicas suelen recibir apoyos dentro de estas aulas y permanecen en ellas la mayor parte del tiempo. En cambio, en los centros ordinarios y el de atención preferente lo reciben en las aulas de PT y AL en momentos puntuales y, en algunos escasos casos, dentro del aula ordinaria. Por tanto, el apoyo sigue siendo mayoritariamente fuera del aula ordinaria y, por ello, no es totalmente compatible con los principios de la escuela inclusiva, que considera que los apoyos deben fomentar la participación con éxito en igualdad de condiciones dentro del aula ordinaria (Arnaiz, 2003) o en contextos normalizados (Muntaner, 2010). Sin embargo, aunque los alumnos con NEE se encuentren dentro del aula ordinaria, puede producirse una exclusión interna: juntos en la misma clase, pero separados por el currículo.

La frecuencia con que se proporciona los apoyos está determinada por la normativa vigente en la Comunidad Valenciana, la cual sigue la mayoría de los centros, aunque su intensidad y duración puede variar en función de las personas y momentos vitales, y deben abarcar todas las facetas de la vida de la persona (Muntaner, 2010). Debe adaptarse a los modos y ritmos de aprendizaje del alumnado y a las dificultades que pueden generar la tarea escolar; hay que racionalizar el tiempo en el aula y que no esté determinado por las materias y por las actividades, sino por la secuenciación lógica de cada grupo de trabajo y por el modo de organizarse para el tema o trabajo a realizar (López Melero, 2001).

El tipo de agrupamiento también está regulado en nuestra comunidad, pero existe un criterio de homogeneidad contrario a los planteamientos inclusivos, que señalan que una de las estrategias de la escuela inclusiva es agrupar a alumnos heterogéneamente y favorecer el aprendizaje cooperativo de los mismos (Arnaiz, 1996, 2011; López Melero, 2011). En las clases heterogéneas, las diferencias entre el alumnado son tenidas en cuenta como algo enriquecedor para el grupo clase y no como un elemento perturbador de la dinámica de trabajo del aula (López Melero, 2001). Observamos que en el aula CyL y en los centros con aulas específicas se establecen grupos y aulas especiales, alejados de un entorno normalizado; en cambio, en el centro ordinario y en el de atención preferente suelen formar agrupamientos en el aula ordinaria o aulas de PT 
Análisis y valoración del modelo inclusivo en los sistemas de apoyo de centros de infantil y primaria

María José Nadal Morant, Claudia Grau Rubio y José Peirats Chacón

y $\mathrm{AL}$, individualmente o en grupos de dos o tres alumnos. Pero, en cualquiera de los casos, se establecen grupos homogéneos en función de las características del alumnado. Por tanto, podemos afirmar que se separa al alumnado en diferentes tipos de agrupamientos: aulas de educación especial, de apoyo, de compensatoria, etc. que implican una reducción en cuanto a sus expectativas de aprendizaje en un contexto ordinario.

Por otra parte, según los resultados del estudio, podemos observar que en el centro ordinario predominan las adaptaciones curriculares basadas en los objetivos y contenidos del aula, frente a las organizativas o de contexto y de acceso al currículo que destacan mayoritariamente en el centro de atención preferente, en el aula CyL, y en el de aulas específicas; ahora bien, en los tres, sobre todo los dos últimos, el alumnado con NEE suele seguir programas específicos en función de sus necesidades, alejados del currículo del aula, lo cual no favorece totalmente la inclusión. Según Sandoval, Simón y Echeita (2012), la mayoría de los profesores entienden las adaptaciones curriculares como programas paralelos a la programación del aula. No se trata de programar y después adaptar, sino contemplar el centro y, en especial, el aula, como un contexto inclusivo a la hora de planificar, tomar decisiones y organizar el proceso de enseñanza-aprendizaje (Moya, 2012). Por otra parte, Ainscow (2001 y 2004) y López Melero (2004) afirman que las adaptaciones curriculares no son la solución y no sólo no han resuelto el problema, sino que hay estudios que ponen de manifiesto que han generado barreras. "Dejemos de crear programas para estudiantes basados en sus etiquetas para pasar a diseñar verdaderos apoyos generalizados" (Wehmeyer, 2009, p. 45). Aunque, ante este argumento, se suele pensar que el nivel educativo puede bajar, pero la realidad es que el currículo de una escuela que apuesta por la inclusión debe ser flexible y creativo, lo cual es sinónimo de más rico y diverso (Darreche, Fernández y Goicoechea, 2010).

Finalmente, si consideramos el tipo de actuaciones de los diferentes profesionales que intervienen en los apoyos, podemos observar que las personas que ejercen apoyo en el centro con aula CyL y en el de unidades específicas suelen trabajar desde un enfoque terapéutico, donde el especialista es un técnico o experto con funciones diferentes del tutor/a. En cambio, en el centro ordinario y el de atención preferente los apoyos colaboran más estrechamente con el tutor/a, pero el alumnado recibe un tratamiento individual o en distintos agrupamientos. Por tanto, todavía sigue imperando el individualismo entre el profesorado. Predomina 
la intervención directa, los especialistas trabajan directamente con el alumnado con NEE, frente a la intervención indirecta, en la que éstos colaboran con los tutores a los que asesoran (Grau, 1998). Cuando intervienen dos profesionales dentro del aula ordinaria (enseñanza cooperativa), según los resultados obtenidos en los cuatro centros, el de apoyo tiene la responsabilidad de integrar al alumnado con NEE en el aula y el tutor/a se responsabiliza del resto. Por tanto, el tipo de actuaciones de los diferentes profesionales no se ajustan totalmente a los planteamientos de la escuela inclusiva.

Existe, por tanto, una visión restrictiva de los modelos de apoyo en cuanto a que se están creando programas, espacios y profesionales diferenciados y segregadores. Se trata de una inclusión aparente o maquillada porque, aunque el de apoyo se encuentra físicamente dentro del aula ordinaria, no existe el mismo nivel de participación de todo el alumnado de la clase, potenciando los programas específicos en función de las necesidades.

\section{Conclusiones}

Atendiendo a los modelos de apoyos internos que presenta Lozano (2007), Moya (2002), Monzón (2011) y Parrilla (1996) señalamos que se ha producido una evolución desde un modelo terapéutico a un apoyo colaborativo-individual; es decir, si anteriormente el apoyo se centraba en el alumno y el maestro de apoyo era un especialista, un experto o un técnico con conocimientos específicos que diseñaba los programas para aplicar a los alumnos con NEE, hoy en día predomina un modelo de apoyo que sigue centrado en el alumno con NEE, aunque con la colaboración de los profesionales de apoyo con el tutor/a.

Sabemos que los enfoques clínicos nos llevan a la discriminación y a la categorización innecesaria del alumnado. En consecuencia, nuestro objetivo debe consistir en acercarnos progresivamente al denominado modelo de apoyo curricular propio de la escuela inclusiva. Un modelo de apoyo generalizado, dirigido a compartir y buscar conjuntamente alternativas educativas que permitan diversificar y adaptar el currículo a todos los alumnos, desde una perspectiva contextual no desde la perspectiva individual-terapéutica.

Por tanto, aunque se observa un cierto avance en el modelo que ac- 
Análisis y valoración del modelo inclusivo en los sistemas de apoyo de centros de infantil y primaria

María José Nadal Morant, Claudia Grau Rubio y José Peirats Chacón

tualmente prevalece en los centros, según los resultados alcanzados se deduce que en las escuelas todavía existe una concepción tradicional del apoyo centrada en el alumno, y ha evolucionado poco el concepto de apoyo centrado en la escuela y en el profesorado. En este sentido, podemos afirmar que queda mucho camino por recorrer, lo que nos empuja a seguir indagando en la realidad de las escuelas para desentrañar la complejidad de las estructuras de apoyo y proponer nuevas líneas de actuación conjuntas, compartidas y contextualizadas.

A la hora de analizar los resultados y sacar conclusiones, no podemos desvincularnos de los condicionantes políticos y sociales que configuran los sistemas de apoyo. Estos factores influyen en el modelo que predomina en los centros reflejados en la normativa vigente. Aunque existe una cierta flexibilidad, consentida desde la legislación actual y desde el centro, se encuentran ciertas limitaciones en algunos elementos de los apoyos que no se ajustan a planteamientos inclusivos. Una de las barreras que impide el aprendizaje y la participación del alumnado en las aulas, y dificultan la construcción de la escuela inclusiva, son las contradicciones que existen en las leyes y normativas respecto a la educación de las personas y culturas diferentes (López Melero, 2011). En consecuencia, las normativas, disposiciones o leyes se van alejando de los principios inclusivos convirtiendo las prácticas pedagógicas en segregadoras.

Desde el análisis y discusión de la información obtenida, podemos concluir que se refleja una aproximación hacia enfoques inclusivos en algunos elementos como son: las personas que proporcionan apoyo, los materiales y recursos didácticos utilizados y la frecuencia con que se proporcionan los apoyos. Sin embargo, existen elementos que todavía se consideran alejados de planteamientos inclusivos como son: el tipo de alumnado que recibe apoyo educativo, el lugar o espacio donde los alumnos reciben los apoyos, el modo de agrupamiento que se establece, el tipo de adaptaciones que se realizan y el tipo de actuaciones de los diferentes profesionales que intervienen en los apoyos.

Para finalizar, consideramos que los sistemas de apoyo instituidos en los centros de Educación Infantil y Primaria de la Comunidad Valenciana, no favorecen de forma completa y generalizada la inclusión educativa del alumnado con NEE. En este sentido, señalamos que no se ha avanzado en la inclusión; se habla mucho de inclusión, pero en realidad no se hace inclusión. Hablar de educación inclusiva requiere cambiar las prácticas pedagógicas, en las que todos sus integrantes forman parte 
de ellas de manera activa y plena y en las que todos se ven implicados en el proceso de inclusión (Torres, 2012; Arnaiz, 2012). Nuestro reto consistirá en establecer un modelo de apoyo, con objeto de mejorar las prácticas inclusivas, que implique medidas organizativas y curriculares adecuadas a las necesidades y características del alumnado del centro. Urge acercarnos hacia un modelo de apoyo curricular centrado en la escuela donde el profesional de apoyo sea considerado como colaborador $y$, al mismo tiempo, corresponsable en el proceso de enseñanza, lejos del apoyo terapéutico centrado en el alumno donde el apoyo es un experto o técnico.

\section{Referencias bibliográficas}

AINSCOW, M. (2001). Desarrollo de escuelas inclusivas. Ideas, propuestas y experiencias para mejorar las instituciones escolares. Madrid: Narcea.

AINSCOW, M. et al. (2004). Investigación-acción. Una propuesta para el desarrollo de prácticas inclusivas. Cuadernos de Pedagogía, 331, 54-59.

ABALDE, E., y MUÑOZ, J. M. (1992). Metodología cuantitativa vs. Cualitativa. Xornadas de Medoloxía de Investigación Educativa. A Coruña: Universidad de La Coruña.

ARNAIZ, P. (1996). Las escuelas son para todos. Siglo Cero, 27(2), 25-34.

ARNAIZ, P. (2003). Educación Inclusiva: una escuela para todos. Málaga: Aljibe.

ARNAIZ, P. (2011). Luchando contra la exclusión: buenas prácticas y éxito escolar. Innovación Educativa, 21, 23-35.

ARNAIZ, P. (2012). Escuelas eficaces e inclusivas: cómo favorecer su desarrollo. Educatio Siglo XXI, 30(1), 25-44.

Asociación Americana de Discapacidades Intelectuales y del Desarrollo (AAIDD) (2011). Discapacidad intelectual. Definición, clasificación y sistemas de apoyo. Madrid: Alianza Editorial.

BOOTH, T. y AINSCOW, M. (2002). Guía para la evaluación y mejora de la educación inclusiva. Madrid: Consorcio universitario para la Educación Inclusiva.

CORONEL, J.M. (1998). Organizaciones escolares. Nuevas propuestas de análisis e investigación. Huelva: Servicio de Publicaciones de la Universidad de Huelva.

DARRECHE, L.; FERNÁNDEZ, A. y GOICOECHEA, J. (2010). ¿La educación inclusiva como utopía que nos ayuda a caminar? Trabajo presentado en el Congreso Iberoamericano de Educación Metas 2021. Buenos Aires, República Argentina.

ESCUDERO, J.M. (2012). La educación inclusiva, una cuestión de derecho. Educatio Siglo XXI, 30(2), 109-128.

ESCUDERO, J.M. y MARTíNEZ, B. (2011). Educación inclusiva y cambio escolar. Revista Iberoamericana de Educación, 55, 85-105.

GINÉ, C. (2001). Inclusión y sistema educativo. Trabajo presentado en III Congreso de 
Análisis y valoración del modelo inclusivo en los sistemas de apoyo de centros de infantil y primaria

María José Nadal Morant, Claudia Grau Rubio y José Peirats Chacón

Atención a la Diversidad en el Sistema Educativo. Instituto Universitario de Integración en la Comunidad (INICO). Salamanca: Universidad de Salamanca.

GOETZ, J.P. y LECOMPTE, M.D. (1988). Etnografía y diseño cualitativo en investigación educativa. Madrid: Morata.

GRAU, C. (1998). Educación especial: De la integración escolar a la escuela inclusiva. Valencia: Promolibro.

LOZANO, J. (2007). Educar en la diversidad. Barcelona: Davinci Continental.

LÓPEZ MELERO, M. (2001). Cortando las amarras de la escuela homogeneizante y segregadora. XXI. Revista de Educación, 3, 15-54.

LÓPEZ MELERO, M. (2004). Construyendo una escuela sin exclusiones. Una forma de trabajar con proyectos en el aula. Málaga: Aljibe.

LÓPEZ MELERO, M. (2011). Barreras que impiden la escuela inclusiva y algunas estrategias para construir una escuela sin exclusiones. Innovación Educativa, 21, 37-54.

MONZÓN, J. (2011). Asesoramiento hacia la escuela inclusiva y formación en centros. Revista interuniversitaria del Profesorado, 70, 111-126.

MORIÑA, A. (2004). Teoría y práctica de la educación inclusiva. Málaga: Ediciones Aljibe.

MOYA, A. (2002). El profesor de apoyo ¿dónde?, ¿cuándo?, ¿cómo? Realiza su trabajo. Málaga: Aljibe.

MOYA, A. (2012). El profesorado de apoyo en los centros ordinarios. Nuevas funciones, nuevas contradicciones. Educatio Siglo XXI, 30(2), 71-88.

MUNTANER, J.J. (2010). De la integración a la inclusión: un nuevo modelo educativo. En P. ARNAIZ, M.D. HURTADO y F.J. SOTO (coords.), 25 Años de Integración Escolar en España: Tecnología e Inclusión en el ámbito educativo, laboral y comunitario (pp. 1-24). Murcia: Consejería de Educación, Formación y Empleo.

NIETO, J.M. (1999). Apoyo educativo a los centros escolares y necesidades especiales. En N. ILLÁN ROMEU (coord.), Didáctica y organización de la educación especial (pp. 109-160). Málaga: Aljibe.

Orden de 16 de julio de 2001 por la que se regula la atención educativa al alumnado con necesidades educativas especiales en centros de Educación Infantil ( $2^{\circ}$ ciclo) y Primaria (DOGV no 4087 de 17.09.2001).

PARRILLA, A. (1992). El profesorado ante la integración escolar: investigación y formación. Argentina: Cincel.

PARRILLA, A. (1996). Apoyo a la escuela: un proceso de colaboración. Bilbao: Mensajero.

SANDOVAL, M.; SIMÓN, C. y ECHEITA, G. (2012). Análisis y valoración crítica de las funciones del profesorado de apoyo desde la educación inclusiva. Revista de educación, número extraordinario, 117-137.

STAINBACK, S. y STAINBACK, W. (1992). Curriculum considerations in inclusive classrooms (faciliting learning for all students). Baltimore: Paul Brookers.

STAINBACK, S. y STAINBACK, W. (1999). Aulas inclusivas. Madrid: Narcea.

STAKE, R.E. (1998). Investigación con estudio de casos. Madrid: Morata.

TAYLOR, S.J. y BOGDAN, R. (1996). Introducción a los métodos cualitativos de investigación. Barcelona, Paidós. 
TORRES, J.A. (2004). La red de apoyo ante las necesidades educativas especiales. En A. Sánchez y J.A. Torres, Educación especial: Centros educativos y profesores ante la diversidad. Madrid: Pirámide.

TORRES, J.A. (2012). Estructuras organizativas para una escuela inclusiva: promoviendo comunidades de aprendizaje. Educatio Siglo XXI, 30(1), 45-70.

WEHMEYER, M. (2009). Autodeterminación y la Tercera Generación de prácticas de inclusión. Revista de Educación, 349, 45-67.

\section{Agradecimientos}

Nuestra gratitud al Programa de Doctorado "Crisis de Legitimidad del Pensamiento y Prácticas Educativas" del Departament de Didàctica i Organització Escolar de la Universitat de València, por la acogida y colaboración recibida, tanto por el profesorado como por el personal de administración y servicios, en la realización de la tesis titulada: "Los sistemas de apoyo en escuelas inclusivas de Educación Infantil y Primaria de la Comunidad Valenciana". 
